\title{
Statyba
}

\section{DUAL MATHEMATICAL MODELS OF LIMIT LOAD ANALYSIS PROBLEMS OF STRUCTURES BY MIXED FINITE ELEMENTS}

\section{S. Kalanta}

To cite this article: S. Kalanta (1997) DUAL MATHEMATICAL MODELS OF LIMIT LOAD ANALYSIS PROBLEMS OF STRUCTURES BY MIXED FINITE ELEMENTS, Statyba, 3:10, 43-51, DOI: $10.1080 / 13921525.1997 .10531683$

To link to this article: https://doi.org/10.1080/13921525.1997.10531683

进 Published online: 26 Jul 2012.

Submit your article to this journal $₫$

Џ Article views: 39 


\section{ДВОЙСТВЕННЫЕ МАТЕМАТИЧЕСКИЕ МОДЕЛИ ЗАДАЧ РАСЧЕТА ПРЕДЕЛЬНОЙ НАГРУЗКИ КОНСТРУКЦИЙ С ПРИМЕНЕНИЕМ СМЕШАННЫХ КОНЕЧНЫХ ЭЛЕМЕНТОВ}

\section{С. Каланта}

\section{1. Введение}

Задачи расчета предельной нагрузки конструкций обычно формулируются в статической и кинематической постановках. Многочисленные исследования по расчету и оптимизации предельной нагрузки континуальных систем (пластин, оболочек и т.д.) с применением конечных элементов можно разделить на две основные групाты (табл. 1). К первой группе отнесем работы [1]-[5] и др., в которьх дискретные математические модели статической и кинематической постановок задач строятся независимо друг от друга с применением соответственно равновесных и совместных конечных элементов. Они позволяют определить нижнюю и верхнюю оценки параметра предельной нагрузки (задачи оптимизации нагрузки не рассматриваются). Возможные разрывы функций скоростей перемещений в поверхностях пластического разрушения конструкщий учитываются только в кинематической постановке задач. При этом кинематическая формулировка задач расчета предельной нагрузки пластинок и оболочек представляет собой задачу невыпуклого программирования. В работах второй групшы ([6]-[9] и др.) применяются равновесные конечные элементы и строятся модели статических постановок задач. Рассматриваются не только задачи определения параметра предельной нагрузки, но и задачи огтимизации. Методом множителей Лагранжа получаются двойственные кинематические постановки. Здесь обе постановки являются задачами выпуклого программирования и позволяют определить нижнюю оценку предельной нагрузки. Однако в них обычно не моделируются поверхности возможных разрывов скоростей перемещений (поверхности пластического разрушения). Поэтому такие постановки задач не могут с достаточной точностью моделировать действительное поведение упруго-пластических пластинок и оболочек, пластическое разрушение которых обычно происходит из-за образования поверхностей сосредоточенных пластических деформаџий, в которых имеют место разрывы функщий скоростей перемещений и диссипация энергии. Нами преследуется цель, используя технику равновесных и совместных конечных элементов, разработать двойственные пары математических моделей задач анализа и огтимизации предельной нагрузки, в которых учитывались бы разрывы функций скоростей перемещений и скорость диссипации энергии в пластических поверхностях конечных элементов (табл. 1, группа III). Двойственные пары моделей на основе равновесных элементов уже построены в [10]. В настоящей статье с использованием совместных конечных элементов и исследований [10], [11] строятся альтернативные двойственные математические модели задач.

Таблица 1. Основные виды задач предельной нагрузки Table 1. Main types of limit load analysis problems

\begin{tabular}{|c|c|c|}
\hline $\begin{array}{l}\text { Группа } \\
\text { работ }\end{array}$ & $\begin{array}{c}\text { Постановки по } \\
\text { равновесию }\end{array}$ & $\begin{array}{c}\text { Постановки по } \\
\text { совместности }\end{array}$ \\
\hline \multirow[b]{2}{*}{ I } & статическая & кинематическая \\
\hline & & \\
\hline \multirow[b]{2}{*}{ II } & статическая & \\
\hline & кинематическая & \\
\hline \multirow[b]{2}{*}{131} & cтатическая & кинтемratическая \\
\hline & knmenamineckins & crammieckar \\
\hline
\end{tabular}




\section{2. Общие вариаџионные постановки задач}

Рассматривается жесткопластическое тело (обобщенная модель конструкции) заданного объема $V$ при известных условиях опирания и функции $C(\mathbf{x})$ параметра текучести материала. На поверхности $S_{f}$ тела распределена внешняя нагрузка $\mathbf{F}(\mathbf{x})$, а на другой части его поверхности $S_{u}$ заданы перемешения $\mathbf{u}(\mathbf{x})=\mathbf{0}$. Определяются напряженно-деформированное состояние (НДС) и параметр $F_{0}$ или оптимальное распределение нагрузкп, отвечающие предельному состоянию тела, возникающему из-за исчерпания его грочности. Тело разделяется на $s$ конечных элеменгов объема $V_{k}, k \in \mathscr{K}$. НЦС $k$-го элемента описывается вектор-функщиями напряжений $\sigma_{k}(\mathbf{x})$, скоростей перемещений $\dot{\mathbf{u}}_{k}(\mathbf{x})$ и деформаций $\dot{\varepsilon}_{k}(\mathbf{x})$. Функции напряжений и скоростей перемещений, относящиеся $\mathrm{K}$ смежным конетным элементам, разделенным поверхностью $S_{t}$, обозначаются знаками “+" и " - ". Пусть номера $t=1,2, \ldots, z$ поверхностей $S_{t}$ конечныл элементов образуют множество

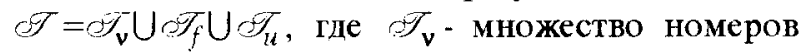
внутренних межэлементных поверхностей; $\mathscr{T}_{f}$ -

множество номеров граничньх, принадлежащих $S_{f}$, поверхностей элементов; $\mathscr{S}_{u} \cdot$ множество номеров граничных поверхностей $S_{u t}$. ПІусть $I_{1}=\pi_{v} \cup T_{f}$.

Математические модели задач анализа и оптимизации предельной нагрузки строятся на основе задач определения стационарной (седловой) точки соответствующих смешанных функционалов. Эти функционалы гголучаются преобразованием представленных в [10] математических моделей статической постановки задач с использованием метода множителей Лагранжа. Итак, задача расчета параметра предельной нагрузки при заданном законе распределения $\mathbf{F}(\mathbf{x})=F_{0} \eta(\mathbf{x})$ представляется задачей стационарHости:

$$
F_{t}=F_{6}+F_{0}\left\{1-\sum_{t \in \mathscr{T}_{t}} \int_{S_{t}} \dot{\mathbf{u}}_{t}^{T}(\mathbf{x}) \eta_{t}(\mathbf{x}) d S_{t}\right\} \Rightarrow \text { stac, (1) }
$$

где

$$
\begin{aligned}
& \mathscr{F}_{0}=\sum_{k} \int_{V_{k}} \sigma_{k}^{T}(\mathbf{x})[£]^{T} \dot{\mathbf{u}}_{k}(\mathbf{x}) d V_{k}+ \\
& +\sum_{t \in \mathscr{O}_{v}} \int_{S_{t}^{-}}\left\{\mathbf{p}_{t}^{-}(\mathbf{x})\right\}^{T}\left\{\dot{\mathbf{u}}_{t}(\mathbf{x})-\dot{\mathbf{u}}_{t}^{-}(\mathbf{x})\right\} d S_{t}+ \\
& +\sum_{t \in \mathbb{O}_{1}} \int_{S_{t}^{+}}\left\{\mathbf{p}_{t}^{+}(\mathbf{x})\right\}^{T}\left\{\dot{\mathbf{u}}_{t}(\mathbf{x})-\dot{\mathbf{u}}_{t}^{+}(\mathbf{x})\right\} d S_{t}- \\
& -\sum_{t \in T_{i}} \int_{S_{i}^{+}}\left\{\mathbf{p}_{t}^{+}(\mathbf{x})\right\}^{T} \dot{\mathbf{u}}_{t}^{+}(\mathbf{x}) d S_{t}+ \\
& +\sum_{k} \int_{V_{k}} \dot{\lambda}_{k}^{T}(\mathbf{x})\left\{\mathbf{f}_{0}\left(C_{k}(\mathbf{x})\right)-\mathbf{f}\left(\sigma_{k}(\mathbf{x})\right)\right\} d V_{k}+ \\
& +\sum_{t \in \mathscr{F}_{v}} \int_{S_{t}^{-}}\left\{\dot{\lambda}_{t}^{-}(\mathbf{x})\right\}^{T}\left\{\mathbf{f}_{0 t}\left(C_{t}^{-}(\mathbf{x})\right)-\mathbf{f}_{t}\left(\mathbf{p}_{t}^{-}(\mathbf{x})\right)\right\} d S_{t}+ \\
& +\sum_{t \in \mathscr{T}} \int_{S_{t}^{+}}\left\{\dot{\lambda}_{t}^{+}(\mathbf{x})\right\}^{T}\left\{\mathbf{f}_{0 t}\left(C_{t}^{+}(\mathbf{x})\right)-\mathbf{f}_{t}\left(\mathbf{p}_{t}^{+}(\mathbf{x})\right)\right\} d S_{t} ; \\
& \dot{\lambda}_{k}(\mathbf{x}) \geq \mathbf{0} \quad \in V_{k} ; \quad \dot{\lambda}_{t}^{-}(\mathbf{x}) \geq \mathbf{0}, \quad \dot{\lambda}_{t}^{+}(\mathbf{x}) \geq \mathbf{0} \in S_{t} .
\end{aligned}
$$

Здесь $F_{0}$ - параметр предельной нагрузки; $[\propto \not \wedge]^{T}$ - оператор дифференциальных геометрических уравнений; $\mathbf{p}_{t}(\mathbf{x}), \dot{\mathbf{u}}_{t}(\mathbf{x})$ - векторы напряжений и скоростей перемещений $t$-й поверхности раздела конечных элементов; $\mathbf{f}\left(\sigma_{k}(\mathbf{x})\right), \mathbf{f}_{t}\left(\mathbf{p}_{t}(\mathbf{x})\right)$ - функции текучести в объеме $V_{k}$ и на поверхности $S_{t}$ конечного элемента; $\dot{\lambda}_{k}(\mathbf{x}), \dot{\lambda}_{t}(\mathbf{x})$ - множители закона пластического течения.

Задача огтимизапии нагрузки при заданном критерии оптимальности $\varphi=\sum_{t \in \mathscr{Y}_{f}} \int_{S_{f t}} \mathbf{T}_{t}^{T}(\mathbf{x}) \mathbf{F}_{t}(\mathbf{x}) d S_{t}$

также может быть представлена задачей стационарности функционала [10]

$\mathscr{F}_{q}=\mathscr{F}_{c}+\sum_{t \in \mathscr{T}_{f}} \int_{S_{j t}} \mathbf{F}_{t}^{T}(\mathbf{x})\left\{\mathbf{T}_{t}(\mathbf{x})-\dot{\mathbf{u}}_{t}(\mathbf{x})+\dot{v}_{t}(\mathbf{x})\right\} d S_{t},(3)$

где множитель $\dot{v}_{t}(\mathbf{x}) \geq 0$.Условие стационарности этих функционалов по статическим переменным $\sigma_{k}(\mathbf{x}), \mathbf{p}_{t}^{-}(\mathbf{x}), \mathbf{p}_{t}^{+}(\mathbf{x})$ и $F_{0}$ или $\mathbf{F}_{t}(\mathbf{x})$ приводит $\mathbf{~}$ следуюшей системе уравнений:

$$
\begin{aligned}
& -\left[\nabla \mathbf{r}\left(\sigma_{k}(\mathbf{x})\right)\right]^{T} \dot{\lambda}_{k}(\mathbf{x})+[\in]^{T} \dot{\mathbf{u}}_{k}(\mathbf{x})=\mathbf{0} \in V_{k}, k \in \mathscr{K} ; \\
& -\left[\nabla \mathbf{f}_{t}\left(\mathbf{p}_{t}^{-}(\mathbf{x})\right)\right]^{T} \dot{\lambda}_{t}^{-}(\mathbf{x})+\dot{\mathbf{u}}_{t}(\mathbf{x})-\dot{\mathbf{u}}_{t}^{-}(\mathbf{x})=\mathbf{0} \in S_{t}^{-}, t \in \mathscr{T}_{\mathbf{v}} ; \\
& -\left[\nabla \mathbf{f}_{t}\left(\mathbf{p}_{t}^{+}(\mathbf{x})\right)\right]^{T} \dot{\lambda}_{t}^{+}(\mathbf{x})+\dot{\mathbf{u}}_{t}(\mathbf{x})-\dot{\mathbf{u}}_{t}^{+}(\mathbf{x})=\mathbf{0} \in S_{t}^{+}, t \in \mathscr{T}_{t} ; \\
& -\left[\nabla \mathbf{f}_{t}\left(\mathbf{p}_{t}^{+}(\mathbf{x})\right)\right]^{T} \dot{\lambda}_{t}^{+}(\mathbf{x})-\dot{\mathbf{u}}_{t}^{+}(\mathbf{x})=\mathbf{0} \in S_{u t}, t \in \mathscr{T}_{u} ; \\
& \dot{\lambda}_{k}(\mathbf{x}) \geq \mathbf{0} \in V_{k}, \quad \dot{\lambda}_{t}^{-}(\mathbf{x}) \geq \mathbf{0} \in S_{t}^{-}, \quad \dot{\lambda}_{t}^{+}(\mathbf{x}) \geq \mathbf{0} \in S_{t}^{+} .
\end{aligned}
$$


$\sum_{t \in \mathscr{O}_{t}} \int_{S_{t}} \dot{\mathbf{u}}_{t}^{T}(\mathbf{x}) \eta_{t}(\mathbf{x}) d S=1 \mid \dot{\mathbf{u}}_{t}(\mathbf{x}) \geq \mathbf{T}_{t}(\mathbf{x}) \in S_{f t}$.

Здесь $\left[\nabla \mathbf{f}\left(\sigma_{k}(\mathbf{x})\right)\right],\left[\nabla \mathbf{f}_{t}\left(\mathbf{p}_{t}(\mathbf{x})\right)\right]$ - матрицы градиентов условий текучести; $\mathbf{T}_{t}(\mathbf{x})$ - вектор-функция весовых коэффициентов критерия оптимальности нагрузки. Система уравнений (4a) представляет собой дифференциальные геометрические уравнения конечных элементов, кинематические условия сопряжения элементов и кинематические граничные условия на поверхности $S_{u}$. Она описывает область кинематически возможныг распределений скоростей перемещений, а условие (5) представляет собой нормализационное ограничение скоростей перемещений и мощности нагрузки для однопараметрической и огтимизационной задач соответственно. Равенство в ограничениях (5) относится $\mathbf{x}$ однопараметрической задаче, а неравенство - $\mathbf{k}$ задаче оптимизации. Зависимости (4a), (5) определяют область кинематически допустимых функций скоростей перемещений.

В случае кинематически допустимых скоростей перемещений функционалы $\mathscr{F}_{f}$ и $\mathscr{F}_{z}$ выражают скорость диссипации энергии конечноэлементной модели. Поэтому задачи стационарности (1) и (3) могут быть преобразованы в задачу минимизации

$$
\begin{aligned}
\mathscr{D}_{t} & =\sum_{k \in \mathscr{K}} \int_{V_{k}} \dot{\lambda}_{k}^{T}(\mathbf{x})\left[\nabla \mathbf{f}\left(\sigma_{k}(\mathbf{x})\right)\right] \sigma_{k}(\mathbf{x}) d V_{k}+ \\
& +\sum_{t \in \mathscr{O}_{v}} \int_{S_{t}}\left\{\dot{\lambda}_{t}^{-}(\mathbf{x})\right\}^{T}\left[\nabla \mathbf{f}_{t}\left(\mathbf{p}_{t}^{-}(\mathbf{x})\right)\right] \mathbf{p}_{t}^{-}(\mathbf{x}) d S_{t}+ \\
& +\sum_{t \in \mathscr{F}} \int_{S_{t}}\left\{\dot{\lambda}_{t}^{+}(\mathbf{x})\right\}^{T}\left[\nabla \mathbf{f}_{t}\left(\mathbf{p}_{t}^{+}(\mathbf{x})\right)\right] \mathbf{p}_{t}^{+}(\mathbf{x}) d S_{t}+\quad(6 \mathbf{a}) \\
& +\sum_{k \in \mathscr{K}} \int_{V_{k}} \dot{\lambda}_{k}^{T}(\mathbf{x})\left\{\mathbf{f}_{0}\left(C_{k}(\mathbf{x})\right)-\mathbf{f}\left(\sigma_{k}(\mathbf{x})\right)\right\} d V_{k}+ \\
& +\sum_{t \in \mathscr{F}_{v}} \int_{S_{t}}\left\{\dot{\lambda}_{t}^{-}(\mathbf{x})\right\}^{T}\left\{\mathbf{f}_{0 t}\left(C_{t}^{-}(\mathbf{x})\right)-\mathbf{f}_{t}\left(\mathbf{p}_{t}^{-}(\mathbf{x})\right)\right\} d S_{t}+ \\
& +\sum_{t \in \mathscr{F}} \int_{S_{t}}\left\{\dot{\lambda}_{t}^{+}(\mathbf{x})\right\}^{T}\left\{\mathbf{f}_{0 t}\left(C_{t}^{+}(\mathbf{x})\right)-\mathbf{f}_{t}\left(\mathbf{p}_{t}^{+}(\mathbf{x})\right)\right\} d S_{t} \Rightarrow \mathbf{m i n}
\end{aligned}
$$
при условиях (4а), (5).

Если на поверхности $S_{t}$ нагрузка не распределена, то $\mathbf{p}_{t}^{+}(\mathbf{x})=-\mathbf{p}_{t}^{-}(\mathbf{x})=\mathbf{p}_{t}(\mathbf{x})$. Поэтому в тех случаях, когда на поверхностях сопряжения конечных элементов нагрузка не приложена, выражение (2а) целесообразно заменить сдедующим более простым выраженикм:

$$
\begin{aligned}
\mathscr{\mathscr { F }}_{O} & =\sum_{k \in \mathscr{O} T} \int_{V_{k}} \sigma_{k}^{T}(\mathbf{x})[\mathscr{S}]^{T} \dot{\mathbf{u}}_{k}(\mathbf{x}) d V_{k}+ \\
& +\sum_{t \in \mathscr{F}_{v}} \int_{S_{t}}\left\{\mathbf{p}_{t}(\mathbf{x})\right\}^{T}\left\{\dot{\mathbf{u}}_{t}^{-}(\mathbf{x})-\dot{\mathbf{u}}_{t}^{+}(\mathbf{x})\right\} d S_{t}+ \\
& +\sum_{t \in \mathscr{T}_{t}} \int_{S_{t}}\left\{\mathbf{p}_{t}(\mathbf{x})\right\}^{T}\left\{\dot{\mathbf{u}}_{t}(\mathbf{x})-\dot{\mathbf{u}}_{t}^{+}(\mathbf{x})\right\} d S_{t}- \\
& -\sum_{t \in \mathscr{F}_{u}} \int_{S_{t}}\left\{\mathbf{p}_{t}(\mathbf{x})\right\}^{T} \dot{\mathbf{u}}_{t}^{+}(\mathbf{x}) d S_{t}+ \\
& +\sum_{k} \int_{V_{k}} \dot{\lambda}_{k}^{T}(\mathbf{x})\left\{\mathbf{f}_{0}\left(C_{k}(\mathbf{x})\right)-\mathbf{f}\left(\sigma_{k}(\mathbf{x})\right)\right\} d V_{k}+ \\
& +\sum_{t \in \mathscr{F}_{v}} \int_{S_{t}} \dot{\lambda}_{t}^{T}(\mathbf{x})\left\{\mathbf{f}_{0 t}\left(C_{t}(\mathbf{x})\right)-\mathbf{f}_{t}\left(\mathbf{p}_{t}(\mathbf{x})\right)\right\} d S_{t} .
\end{aligned}
$$

Тогда седловая точка функционалов определяется системой уравнений

$$
\begin{aligned}
& -\left[\nabla \mathbf{f}\left(\sigma_{k}(\mathbf{x})\right)\right]^{T} \dot{\lambda}_{k}(\mathbf{x})+[\mathscr{A}]^{T} \dot{\mathbf{u}}_{k}(\mathbf{x})=\mathbf{0} \in V_{k}, k \in \mathscr{O} ; \\
& -\left[\nabla \mathbf{f}_{t}\left(\mathbf{p}_{t}(\mathbf{x})\right)\right]^{T} \dot{\lambda}_{t}(\mathbf{x})+\dot{\mathbf{u}}_{t}^{-}(\mathbf{x})-\dot{\mathbf{u}}_{t}^{+}(\mathbf{x})=\mathbf{0} \in S_{t}, \quad t \in \mathscr{O}_{\mathbf{v}} ; \\
& -\left[\nabla \mathbf{f}_{t}\left(\mathbf{p}_{t}(\mathbf{x})\right)\right]^{T} \dot{\lambda}_{t}(\mathbf{x})+\dot{\mathbf{u}}_{t}(\mathbf{x})-\dot{\mathbf{u}}_{t}^{+}(\mathbf{x})=\mathbf{0} \in S_{t}, \quad t \in \mathscr{O}_{f} ; \\
& -\left[\nabla \mathbf{f}_{t}\left(\mathbf{p}_{t}(\mathbf{x})\right)\right]^{T} \dot{\lambda}_{t}(\mathbf{x})-\dot{\mathbf{u}}_{t}^{+}(\mathbf{x})=\mathbf{0} \in S_{u t}, \quad t \in \mathscr{O}_{u} ; \\
& \dot{\lambda}_{k}(\mathbf{x}) \geq \mathbf{0} \in V_{k}, \quad \dot{\lambda}_{t}(\mathbf{x}) \geq \mathbf{0} \in S_{t}, \quad t \in \mathscr{T} ; \\
& \sum_{t \in \mathscr{O}_{f}} \int_{S_{t}} \dot{\mathbf{u}}_{t}^{T}(\mathbf{x})_{t}(\mathbf{x}) d S=1 \text { или } \dot{\mathbf{u}}_{t}(\mathbf{x}) \geq \mathbf{T}_{t}(\mathbf{x}) \in S_{f t},(5)
\end{aligned}
$$

эквивалентной системе (4a), (5). В этом случае задачи стационарности (1), (3) и задача минимизации (6а) преобразуются в задачу

$$
\begin{aligned}
& \dot{\mathscr{D}}_{z}=\sum_{k \in \mathscr{K}} \int_{V_{k}} \dot{\lambda}_{k}^{T}(\mathbf{x})\left[\nabla \mathbf{f}\left(\sigma_{k}(\mathbf{x})\right)\right] \sigma_{k}(\mathbf{x}) d V_{k}+ \\
& \quad+\sum_{t \in \mathscr{T}} \int_{S_{t}} \dot{\lambda}_{t}^{T}(\mathbf{x})\left[\nabla \mathbf{f}_{t}\left(\mathbf{p}_{t}(\mathbf{x})\right)\right] \mathbf{p}_{t}(\mathbf{x}) d S_{t}+ \\
& \quad+\sum_{k \in \mathscr{K} \mathscr{K}} \int_{V_{k}} \dot{\lambda}_{k}^{T}(\mathbf{x})\left\{\mathbf{f}_{0}\left(C_{k}(\mathbf{x})\right)-\mathbf{f}\left(\sigma_{k}(\mathbf{x})\right)\right\} d V_{k}+ \\
& +\sum_{t \in \mathscr{T} T} \int_{S_{t}} \dot{\lambda}_{t}^{T}(\mathbf{x})\left\{\mathbf{p}_{0 t}\left(C_{t}(\mathbf{x})\right)-\mathbf{f}_{t}\left(\mathbf{p}_{t}(\mathbf{x})\right)\right\} d S_{t} \Rightarrow \mathbf{m i n}
\end{aligned}
$$

при условиях (46), (5). Три первых члена функционала $\dot{\mathscr{X}}_{f}$, а также два первых члена функционала $\dot{\mathscr{D}}_{2}$ выражают скорость диссипации энергии в объеме конечных элементов и в межэлементных поверхностях, а остальные члены для оптимального рештения задач (6) равны нулю. Поэтому задачи (6) отвечают 
кинематической теореме предельной нагрузки о минимуме скорости диссипации энергии [12], [13]. Когда внешняя нагрузка распределена лишь на некоторьх поверхностях сопряжения конечных элементов, можно применять гибридные функционалы и условия сопряжения: для загруженньх поверхностей - зависимости (2a), (4a), а для остальных - (26), (46).

\section{3. Дискретные математические модели} кинематической постановки задач

Дэя дискретизации задач (1), (3) или (6) применяются смешанные конечные элементы. Выбираются интерполяционные функции

$$
\begin{array}{ll}
\sigma_{k}(\mathbf{x})=\left[H_{k}(\mathbf{x})\right] \sigma_{k}, & \mathbf{p}_{t}(\mathbf{x})=\left[H_{t}(\mathbf{x})\right] \mathbf{p}_{t} ; \\
\dot{\lambda}_{k}(\mathbf{x})=\left[H_{\lambda k}(\mathbf{x})\right] \dot{\lambda}_{k}, & \dot{\lambda}_{t}(\mathbf{x})=\left[H_{\lambda t}(\mathbf{x})\right] \dot{\lambda}_{t} ; \\
\dot{\mathbf{u}}_{k}(\mathbf{x})=\left[H_{u k}(\mathbf{x})\right] \dot{\mathbf{u}}_{k}, & \dot{\mathbf{u}}_{t}(\mathbf{x})=\left[H_{u t}(\mathbf{x})\right] \mathbf{u}_{t} ; \\
C_{k}(\mathbf{x})=\left[H_{0 k}(\mathbf{x})\right] \mathbf{C}_{k} ; & \mathbf{F}_{t}(\mathbf{x})=\left[H_{f t}(\mathbf{x})\right] \mathbf{F}_{t} .
\end{array}
$$

Здесь $\sigma_{k}, \dot{\mathbf{u}}_{k}, \dot{\lambda}_{k}, \mathbf{C}_{k}$ - векторы напряжений, скоростей перемещений, пластических множите.тей и параметров текучести узловых точек $k$-го элемента. Функщии $\sigma_{k}(\mathbf{x})$ и $\dot{\mathbf{u}}_{k}(\mathbf{x})$ должны быть построены так, ттобы при равенстве обобщенных узловых перемещений $\dot{\mathbf{u}}_{k}$ и напряжений $\sigma_{k}$ смежньх конечньх элементов соблюдалась неразрывность функций (7) и другие требования сходимости [14]

После подстановки функций (7) задачи (1), (3) получают следующий дискретный вид:

а) однопараметрическая задача -

$\widetilde{F}_{t d}=\sum_{k \in \mathscr{K}} \sigma_{k}^{T}\left[A_{k}\right]^{T} \dot{\mathbf{u}}_{k}+\sum_{t \in \mathscr{F}_{v}}\left\{\mathbf{p}_{t}^{-}\right\}^{T}\left[A_{t}\right]^{T}\left(\dot{\mathbf{u}}_{t}-\dot{\mathbf{u}}_{t}^{-}\right)+$

$+\sum_{t \in \mathcal{T}_{1}}\left\{\mathbf{p}_{t}^{+}\right\}^{T}\left[A_{t}\right]^{T}\left(\dot{\mathbf{u}}_{t}-\dot{\mathbf{u}}_{t}^{+}\right)-\sum_{t \in \mathscr{F}_{\mathcal{T}}}\left\{\mathbf{p}_{t}^{+}\right\}^{T}\left[A_{t}\right]^{T} \dot{\mathbf{u}}_{t}^{+}+$

$+\sum_{k \in \mathcal{K}} \dot{\lambda}_{k}^{T}\left\{\mathbf{f}_{0 k}\left(\mathbf{C}_{k}\right)-\mathbf{f}_{k}\left(\sigma_{k}\right)\right\}+$

$+\sum_{t \in \mathscr{F}_{v}}\left\{\dot{\lambda}_{t}^{-}\right\}^{T}\left\{\mathbf{f}_{0 t}\left(\mathbf{C}_{t}^{-}\right)-\mathbf{f}_{t}\left(\mathbf{p}_{t}^{-}\right)\right\}+$

$+\sum_{t \in \mathscr{T}_{1}}\left\{\dot{\lambda}_{t}^{+}\right\}^{T}\left\{\mathbf{f}_{0 t}\left(\mathbf{C}_{t}^{+}\right)-\mathbf{f}_{t}\left(\mathbf{p}_{t}^{+}\right)\right\}+F_{0}-F_{0} \sum_{t \in \mathscr{C}_{f}} \dot{\mathbf{u}}_{f t}^{T} \boldsymbol{\eta}_{t}=$ $=\mathscr{F}_{c d}+F_{0}-F_{0} \sum_{t \in \mathscr{C}_{f}} \dot{\mathbf{u}}_{f t}^{T} \eta_{t} \Rightarrow$ stac; $\quad \dot{\lambda}_{k} \geq \mathbf{0}, \quad \dot{\lambda}_{t} \geq \mathbf{0}$ б) задача оптимизации нагрузки -

$$
\begin{gathered}
\mathscr{T}_{2 d}=\mathscr{F}_{\text {td }}+\sum_{t \in \mathscr{T},} \mathbf{F}_{t}^{T}\left\{\mathbf{T}_{t}-\left[V_{t}\right] \dot{\mathbf{u}}_{t}+\dot{v}_{t}\right\} \Rightarrow \text { stac } ; \\
\dot{\lambda}_{k} \geq \mathbf{0}, \quad \dot{\lambda}_{t}^{-} \geq \mathbf{0}, \quad \dot{\lambda}_{t}^{+} \geq \mathbf{0} .
\end{gathered}
$$

Здесь векторы критерия оптимальности и распределения нагрузки

$$
\begin{aligned}
& \mathbf{T}_{t}=\int_{S_{f t}}\left[H_{f t}(\mathbf{x})\right]^{T} \mathbf{T}_{t}(\mathbf{x}) d S_{t}, \dot{v}_{t}=\int_{S_{f}}\left[H_{f t}(\mathbf{x})\right]^{T} \dot{v}_{t}(\mathbf{x}) d S_{t}, \\
& \eta_{t}=\int_{S_{f t}}\left[H_{u t}(\mathbf{x})\right]^{T} \eta_{t}(\mathbf{x}) d S_{t},
\end{aligned}
$$

а также функщий текучести конечного элемента

$$
\begin{aligned}
& \mathbf{f}_{k}\left(\sigma_{k}\right)=\int_{V_{k}}\left[H_{\lambda k}(\mathbf{x})\right]^{T} \mathbf{f}\left(\sigma_{k}(\mathbf{x})\right) d V_{k}, \\
& \mathbf{f}_{0 k}\left(\mathbf{C}_{k}\right)=\int_{V_{k}}\left[H_{\lambda k}(\mathbf{x})\right]^{T} \mathbf{f}_{0}\left(C_{k}(\mathbf{x})\right) d V_{k}, \\
& \mathbf{f}_{t}\left(\mathbf{p}_{t}\right)=\int_{S_{t}}\left[H_{\lambda t}(\mathbf{x})\right]^{T} \mathbf{f}_{t}\left(\mathbf{p}_{t}(\mathbf{x})\right) d S_{t}, \\
& \mathbf{f}_{0 t}\left(\mathbf{C}_{t}\right)=\int_{S_{t}}\left[H_{\lambda t}(\mathbf{x})\right]^{T} \mathbf{f}_{0 t}\left(C_{t}(\mathbf{x})\right) d S_{t} .
\end{aligned}
$$

Матрицы

$$
\begin{aligned}
& {\left[A_{k}\right]^{T}=\int_{V_{k}}\left[H_{k}(\mathbf{x})\right]^{T}[\cong \mathcal{A}]^{T}\left[H_{u k}(\mathbf{x})\right] d V_{k},} \\
& {\left[A_{t}\right]^{T}=\int_{S_{t}}\left[H_{t}(\mathbf{x})\right]^{T}\left[H_{u t}(\mathbf{x})\right] d S_{t},} \\
& {\left[V_{t}\right]=\int_{S_{t}}\left[H_{f t}(\mathbf{x})\right]^{T}\left[H_{u t}(\mathbf{x})\right] d S_{t} .}
\end{aligned}
$$

Используя зависимость (26), можно построить второе - сокращенное выражение функционала

$$
\begin{aligned}
& \mathscr{F}_{C d}=\sum_{k \in \in K^{T}} \sigma_{k}^{T}\left[A_{k}\right]^{T} \dot{\mathbf{u}}_{k}+\sum_{t \in \mathscr{T}_{v}} \mathbf{p}_{t}^{T}\left[A_{t}\right]^{T}\left(\dot{\mathbf{u}}_{t}^{-}-\dot{\mathbf{u}}_{t}^{+}\right)+ \\
& +\sum_{t \in \mathscr{F}_{t}} \mathbf{p}_{t}^{T}\left[A_{t}\right]^{T}\left(\dot{\mathbf{u}}_{t}-\dot{\mathbf{u}}_{t}^{+}\right)-\sum_{t \in \mathscr{F}_{t}} \mathbf{p}_{t}^{T}\left[A_{t}\right]^{T} \dot{\mathbf{u}}_{t}^{+}+ \\
& +\sum_{k} \dot{\lambda}_{k}^{T}\left\{\mathbf{f}_{0 k}\left(\mathbf{C}_{k}\right)-\mathbf{f}_{k}\left(\sigma_{k}\right)\right\}+\sum_{t} \dot{\lambda}_{t}^{T}\left\{\mathbf{f}_{0 t}\left(\mathbf{C}_{t}\right)-\mathbf{f}_{t}\left(\mathbf{p}_{t}\right)\right\}
\end{aligned}
$$

и соответственно преобразовать функционалы $\mathscr{F}_{r d}, \mathscr{F}_{2 d}$. ЭТи сокращенные выражения можно использовать в тех случаях, когда на поверхностях сопряжения конечных элементов нагрузка не ггриложена.

Условие стационарности функционала $\mathscr{F}_{\text {t }}$ или $F_{2 d}$ по переменным $\sigma_{k}$ и $\mathbf{p}_{t}^{-}, \mathbf{p}_{t}^{+}, F_{0}$ или 
$\mathbf{p}_{t}, F_{t}$ позволяет получить дискретные выражения геометрических уравнений конечных элементов

$-\left[\nabla \mathbf{f}_{k}\left(\sigma_{k}\right)\right]^{T} \dot{\lambda}_{k}+\left[A_{k}\right]^{T} \dot{\mathbf{u}}_{k}=\mathbf{0}, \quad \dot{\lambda}_{k} \geq \mathbf{0}, \quad k \in \varrho \mathscr{K}$,

устовий совместности скоростей перемещений между элементами

$$
\begin{aligned}
& -\left[\nabla \mathbf{f}_{t}\left(\mathbf{p}_{t}^{-}\right)\right]^{T} \dot{\lambda}_{t}^{-}+\left[A_{t}\right]^{T}\left(\dot{\mathbf{u}}_{t}-\dot{\mathbf{u}}_{t}^{-}\right)=\mathbf{0}, \quad \dot{\lambda}_{t}^{-} \geq \mathbf{0}, \quad t \in \mathscr{F}_{\mathbf{v}} ; \\
& -\left[\nabla \mathbf{f}_{t}\left(\mathbf{p}_{t}^{+}\right)\right]^{T} \dot{\lambda}_{t}^{+}+\left[A_{t}\right]^{T}\left(\dot{\mathbf{u}}_{t}-\dot{\mathbf{u}}_{t}^{+}\right)=\mathbf{0}, \quad \dot{\lambda}_{t}^{+} \geq \mathbf{0}, \quad t \in \mathscr{T}_{1}
\end{aligned}
$$

ити

$$
-\left[\nabla \mathbf{f}_{t}\left(\mathbf{p}_{t}\right)\right]^{T} \dot{\lambda}_{t}+\left[A_{t}\right]^{T}\left(\dot{\mathbf{u}}_{\bar{t}}-\dot{\mathbf{u}}_{t}^{+}\right)=\mathbf{0}, \dot{\lambda}_{t} \geq \mathbf{0}, t \in \mathscr{F}_{1},
$$

кинематических граничных условий

$$
-\left[\nabla \mathbf{f}_{t}\left(\mathbf{p}_{t}^{+}\right)\right]^{T} \dot{\lambda}_{t}^{+}-\left[A_{t}\right]^{T} \dot{\mathbf{u}}_{t}^{+}=\mathbf{0}, \quad \dot{\lambda}_{t}^{+} \geq \mathbf{0}, \quad t \in \mathscr{T}_{u}
$$

и условий нормализации

$$
\sum_{t \in \mathcal{O}_{:}} \dot{\mathbf{u}}_{f t}^{T} \eta_{t}=1 \quad \text { ити } \quad \mathbf{T}_{t}+\dot{\mathbf{v}}_{t}-\left[V_{t}\right] \dot{\mathbf{u}}_{f t}=\mathbf{0}, \dot{\mathbf{v}}_{t} \geq \mathbf{0}
$$

Объединяя и принимая их за предварительные условия, задачи определения стационарной точки функционатов $\widetilde{F}_{k t}$ и $\mathscr{F}_{2 d}$ Iреобразуются в следующие задачи минимизации:

а) задача определения параметра предельной нагрузки -

$$
\begin{aligned}
\dot{\mathscr{Y}} & =\dot{\lambda}_{\sigma}^{T}\left[\nabla \mathbf{f}_{\boldsymbol{\sigma}}(\sigma)\right] \sigma+\dot{\lambda}_{p}^{T}\left[\nabla \mathbf{f}_{p}(\mathbf{p})\right] \mathbf{p}+ \\
& +\dot{\lambda}_{\sigma}^{T}\left\{\mathbf{f}_{0 \sigma}(\mathbf{C})-\mathbf{f}_{\sigma}(\sigma)\right\}+\dot{\lambda}_{p}^{T}\left\{\mathbf{f}_{0 p}(\mathbf{C})-\mathbf{f}_{p}(\mathbf{p})\right\} \Rightarrow \text { min }
\end{aligned}
$$

при условиях

$$
\begin{aligned}
& -\left[\nabla \mathbf{f}_{\sigma}(\sigma)\right]^{T} \dot{\lambda}_{\sigma}+\left[A_{\sigma}\right]^{T} \dot{\mathbf{u}}=\mathbf{0}, \quad \dot{\lambda}_{\sigma} \geq \mathbf{0}, \\
& -\left[\nabla \mathbf{f}_{p}(\mathbf{p})\right]^{T} \dot{\lambda}_{p}+\left[A_{p}\right]^{T} \dot{\mathbf{u}}=\mathbf{0}, \quad \dot{\lambda}_{p} \geq \mathbf{0}, \quad \dot{\mathbf{u}}^{T} \eta=1 ;
\end{aligned}
$$

6) задача огтимизации нагрузки -

$$
\text { найти } \quad \dot{\Phi} \Rightarrow \min
$$

при условиях

$$
\begin{aligned}
& -\left[\nabla \mathbf{f}_{\sigma}(\sigma)\right]^{T} \dot{\lambda}_{\sigma}+\left[A_{\sigma}\right]^{T} \dot{\mathbf{u}}=\mathbf{0}, \quad \dot{\lambda}_{\boldsymbol{\sigma}} \geq \mathbf{0}, \\
& -\left[\nabla \mathbf{f}_{p}(\mathbf{p})\right]^{T} \dot{\lambda}_{p}+\left[A_{p}\right]^{T} \dot{\mathbf{u}}=\mathbf{0}, \quad \dot{\lambda}_{p} \geq \mathbf{0}, \quad[V] \mathbf{u} \geq \mathbf{T} .
\end{aligned}
$$

Здесь $\left[\bar{A}_{\boldsymbol{\sigma}}\right],\left[\nabla \mathbf{f}_{\boldsymbol{\sigma}}(\sigma)\right],\left[\nabla \mathbf{f}_{p}(\mathbf{p})\right]$ - алгебраические матрицы, диагональными блоками которьх являются матрицы $\left[A_{k}\right],\left[\nabla \mathbf{f}_{k}\left(\sigma_{k}\right)\right],\left[\nabla \mathbf{f}_{t}\left(\mathbf{p}_{t}\right)\right]$. Матрица $\left[A_{\sigma}\right]^{T} \equiv\left[\left[\bar{A}_{\sigma}\right]^{T},[0]\right]$ получается добавлением нулевых столбцов к матрице $\left[\bar{A}_{\sigma}\right]^{T}$. Векторы $\sigma, \dot{\lambda}$ состоят из совокупности векторов $\sigma_{k}, \dot{\lambda}_{k}, k=1,2, \ldots, s$. Состав.ляюшими векторов $\mathbf{p}, \lambda_{p}, \dot{\mathbf{u}}$ яв.тяются векторы $\mathbf{p}_{t}^{-}, \mathbf{p}_{t}^{+}, \dot{\lambda}_{t}^{-}, \dot{\lambda}_{t}^{+}$и $\dot{\mathbf{u}}_{k}, \dot{\mathbf{u}}_{t}$ соответственно.

Неизвестными в задачах (14) и (15) являются векторы напряжений, скоростей перемещений и пластических множителей. Однако вектор і можно исключить из числа основных неизвестных. Вводим векторы $\bar{\sigma} \equiv\{\sigma, \mathbf{p}\}^{T}, \dot{\lambda} \equiv\left\{\dot{\lambda}_{\boldsymbol{\sigma}}, \dot{\lambda}_{p}\right\}^{T}, \mathbf{f}(\bar{\sigma}) \equiv\left\{\mathbf{f}_{\sigma}(\sigma), \mathbf{f}_{p}(\mathbf{p})\right\}^{T}$, $\mathbf{f}_{0}(\mathbf{C}) \equiv\left\{\mathbf{f}_{0 \sigma}(\mathbf{C}), \mathbf{f}_{0 p}(\mathbf{C})\right\}^{T}$. Тогда геометрические уравнения можно представить одним матричным уравнением

$$
-[\nabla \mathbf{f}(\bar{\sigma})]^{T} \dot{\lambda}+[A]^{T} \dot{\mathbf{u}}=\mathbf{0}, \quad \dot{\lambda} \geq \mathbf{0}
$$

и задачи (14), (15) записать в сокращенном виде: найти

$$
\dot{\mathscr{Q}}=\dot{\lambda}^{T}[\nabla \mathbf{f}(\bar{\sigma})] \bar{\sigma}+\dot{\lambda}^{T}\left\{\mathbf{f}_{0}(\mathbf{C})-\mathbf{f}(\bar{\sigma})\right\} \Rightarrow \min
$$

при условиях

$$
\begin{array}{c|c}
-[\nabla \mathbf{f}(\bar{\sigma})]^{T} \dot{\lambda}+[A]^{T} \dot{\mathbf{u}}=\mathbf{0}, \quad \dot{\lambda} \geq \mathbf{0}, \\
\dot{\mathbf{u}}^{T} \eta=1 & {[V] \dot{\mathbf{u}} \geq \mathbf{T} .}
\end{array}
$$

Здесь левое условие нормализации относится к однопараметрической задаче, а правое - к задаче огтимизации. Матрицы

$$
[\nabla \mathbf{f}(\bar{\sigma})]=\left[\begin{array}{r}
{\left[\nabla \mathbf{f}_{\sigma}(\sigma)\right]} \\
\left.\left[\nabla \mathbf{f}_{p}(\mathbf{p})\right]\right], \quad[A]=\left[\left[A_{\sigma}\right],\left[A_{p}\right]\right] .
\end{array}\right.
$$

Выбирая базисную матрицу $\left[A_{1}\right]^{T}$, уравнение (16) записываем в вице двух уравнений:

$$
\begin{aligned}
& -\left[\nabla \mathbf{f}_{1}(\bar{\sigma})\right]^{T} \dot{\lambda}+\left[A_{1}\right]^{T} \dot{\mathbf{u}}=\mathbf{0}, \\
& -\left[\nabla \mathbf{f}_{2}(\bar{\sigma})\right]^{T} \dot{\lambda}+\left[A_{2}\right]^{T} \dot{\mathbf{u}}=\mathbf{0} .
\end{aligned}
$$

Решение первого уравнения

$$
\dot{\mathbf{u}}=\left(\left[A_{1}\right]^{T}\right)^{-1}\left[\nabla \mathbf{f}_{1}(\bar{\sigma})\right]^{T} \dot{\lambda}=\left[B_{u}(\bar{\sigma})\right] \dot{\lambda}
$$


подстав:тяется во второе уравнение. Голучается уравнение совместности деформаций

$$
[B(\bar{\sigma})] \dot{\lambda}=\mathbf{0},
$$

Где

$$
[B(\bar{\sigma})]=\left[A_{2}\right]^{T}\left[B_{u}(\bar{\sigma})\right]-\left[\nabla \mathbf{f}_{2}(\bar{\sigma})\right]^{T} \text {. }
$$

С учетом зависимостей (18) и (19) математическая модель кинематической постановки задач (17) модифицируется к вцду:

найาи

$$
\dot{\mathscr{C}}=\dot{\lambda}^{T}[\nabla \mathbf{f}(\bar{\sigma})] \bar{\sigma}+\dot{\lambda}^{T}\left\{\mathbf{f}_{0}(\mathbf{C})-\mathbf{f}(\bar{\sigma})\right\} \Rightarrow \mathbf{m i n}
$$

при условиях

$$
\begin{gathered}
{[B(\bar{\sigma})] \dot{\lambda}=\mathbf{0}, \quad \dot{\lambda} \geq \mathbf{0},} \\
\dot{\lambda}^{T}\left[B_{u}(\bar{\sigma})\right]^{T} \eta=1 \quad[V]\left[B_{u}(\bar{\sigma})\right] \dot{\lambda} \geq \mathbf{T} .
\end{gathered}
$$

Решентем задачи (20) являются векторы $\bar{\sigma}^{*}, \dot{\lambda}^{*}$ I величина скорости диссипации энергии $\dot{\mathscr{D}}^{*}$. Зависимость (18) позволяет исключить скорости перемещений из числа основных неизвестных и определить вектор і после решения задачи (20).

В частньх спучаях условий текучести появляются и другие возможности уменьшения числа основных неизвестных. Например, в случае линейных условий текучести

$$
\mathbf{f}(\bar{\sigma})=[\Phi] \bar{\sigma} \leq \mathbf{C}_{0}=\mathbf{f}_{0}(\mathbf{C})
$$

математические модели (17) и (20) принимают с.тедующий вид:

$$
\text { а) найти } \quad \mathbf{C}_{0}^{T} \dot{\lambda} \Rightarrow \min
$$

прн условиях

$$
\begin{gathered}
-[\Phi]^{T} \dot{\lambda}+[A]^{T} \dot{\mathbf{u}}=\mathbf{0}, \quad \dot{\lambda} \geq \mathbf{0}, \\
\dot{\mathbf{u}}^{T} \eta=1 \quad[V] \dot{\mathbf{u}} \geq \mathbf{T} ;
\end{gathered}
$$

б) найти $\quad \mathrm{C}_{0}^{T} \dot{\lambda} \Rightarrow \min$

при услювиях

$$
\begin{gathered}
{[B] \dot{\lambda}=\mathbf{0}, \quad \dot{\lambda} \geq \mathbf{0},} \\
\dot{\lambda}^{T}\left[B_{u}\right]^{T} \eta=1
\end{gathered}
$$

где неизвестным является лишь вектор пластических множителей, а матрицы

$\left[B_{u}\right]=\left(\left[A_{1}\right]^{T}\right)^{-1}\left[\Phi_{1}\right]^{T},[B]=\left[A_{2}\right]^{T}\left[B_{u}\right]-\left[\Phi_{2}\right]^{T}$.
В случае линейных условий текучести достаточно задать интерполяционные функции лишь для скоростей перемещений, пластических множителей и распределенной нагрузки, т.е. применять геометрически совместные конечные элементы.

\section{4. Дискретные математические модели статической постановки задач}

С использованием метода множителей Лагранжа кинематические постановки задач могут быть преобразованы в двойственные $\mathrm{K}$ ним статические постановки.

Задача определения параметра нагрузки $F_{0}$. Преобразованием задачи (14) получается следуюшая математическая модель:

$$
\text { найти } \quad F_{0} \Rightarrow \max
$$

при условичх

$$
\begin{aligned}
& \mathbf{f}_{\boldsymbol{\sigma}}(\sigma) \leq \mathbf{f}_{0 \boldsymbol{\sigma}}(\mathbf{C}), \quad \mathbf{f}_{p}(\mathbf{p}) \leq \mathbf{f}_{0 p}(\mathbf{C}), \\
& -\left[A_{\boldsymbol{\sigma}}\right] \sigma-\left[A_{p}\right] \mathbf{p}+\eta F_{0}=\mathbf{0}
\end{aligned}
$$

С учетом принятых выпе обозначений она трансформируется в задачу:

$$
\text { найти } \quad F_{0} \Rightarrow \max
$$

при условиях

$$
\mathbf{f}(\bar{\sigma}) \leq \mathbf{f}_{0}(\mathbf{C}), \quad-[A] \bar{\sigma}+\eta F_{0}=\mathbf{0},
$$

которая двойственна задаче (17). Ограничениянеравенства в условиях задач (21) и (22) представляют собой условия текучести, а равенства - уравнения равновесия дискретной модели конструкции. Вместе они определяют множество статически допустимьх векгоров напряжений $\bar{\sigma}$. Таким образом, задачи (21), (22) отвечают известной теореме А.Гвоздева о множите.те предельной нагрузки.

Используя решение уравнений равновесия

$$
\bar{\sigma}=\left[B_{1}\right] \eta F_{0}+\left[B_{2}\right] \bar{\sigma}_{2},
$$

можно исключить базисные напряжения $\bar{\sigma}_{1}$. Здесь $\left[B_{1}\right],\left[B_{2}\right]$ - матрицы частного и общего решений уравнений равновесия [15]. Тогда погучается задача: 
найти

$F_{0} \Rightarrow \max$ при условиях $\overline{\mathbf{f}}\left(\bar{\sigma}_{2}, F_{0}\right) \leq \mathbf{f}_{0}(\mathbf{C})$.

Эта модель двойственна задаче (20) и также может быть получена преобразованием последней при помоци метода множителей Лагранжа.

Задача оптимизации нагрузки. Результатом применения преобразований Јагранжа к задачам (15), (17) и (20) являются следующие математические модели статической постановки задачи оптимизации нагрузки:
a) найти

$$
\mathbf{T}^{T} \mathbf{F} \Rightarrow \mathbf{m a x}
$$

при условиях

$$
\begin{gathered}
\mathbf{f}_{\boldsymbol{\sigma}}(\sigma) \leq \mathbf{f}_{0 \boldsymbol{\sigma}}(\mathbf{C}), \quad \mathbf{f}_{p}(\mathbf{p}) \leq \mathbf{f}_{0 p}(\mathbf{C}), \\
-\left[A_{\boldsymbol{\sigma}}\right] \sigma-\left[A_{p}\right] \mathbf{p}+[V]^{T} \mathbf{F}=\mathbf{0}, \quad \mathbf{F} \geq \mathbf{0} ;
\end{gathered}
$$

6) найти $\quad \mathbf{T}^{T} \mathbf{F} \Rightarrow \max$

при условиях

$$
\mathbf{f}(\bar{\sigma}) \leq \mathbf{f}_{0}(\mathbf{C}), \quad-[A] \bar{\sigma}+[V]^{T} \mathbf{F}=\mathbf{0}, \quad \mathbf{F} \geq \mathbf{0} ;
$$

в) найти

$$
\mathbf{T}^{T} \mathbf{F} \Rightarrow \mathbf{m a x}
$$

при условиях $\quad \overline{\mathbf{f}}\left(\bar{\sigma}_{2}, \mathbf{F}\right) \leq \mathbf{f}_{0}(\mathbf{C}), \quad \mathbf{F} \geq \mathbf{0}$.

При фиксированных скоростях перемещений $\dot{\mathbf{u}}=\mathbf{T}$ функщия цели этих задач имеет физический смысл мощности внешней нагрузки. Поэтому задачи (24)-(26) отвечают статической теореме предельной нагрузки (о мощности нагрузки [12], \{16]).

\section{5. Заключение}

Разработанные математические модели гредставляют собой двойсгвенные пары задач математического программирования в кинематической и статической формулировках. Они позволяют определять верхние оценки напряжений, скоростей перемещений и пластических множителей, скорости диссипации энергии, мощности нагрузки и параметра $F_{0}$ или оптимального распределения нагрузки. Кинематические постановки являются прямыми задачами. Онгі позволяют определить распределение скоростей перемещений, птастических множителей и напряжений (только в случае нелинейных условий текучести) и величину скорости диссипации энергии. Скорости перемещений и пластических множителей (кинематические величины) характеризуют механизм пластического разрушения конструкщии. Однако для инженерной практики наиболее важны статические величины, т.е. распределение напряжений и предельной нагрузки. Они определяются решением двойственных задач - статических математических моделей (21) - (26).

Согласно первой теореме двойственности для оптималыных решений задач (14) и (21) $F_{0}^{*}=\dot{\mathscr{D}}^{*}$. Поэтому решением кинематических постановок (14), (20) определяется и значение параметра разрушаюшей нагрузки. Однако решение кинематической постановки задачи огтимизации не дает прямого ответа об огтимальном распределении нагрузки. В случае нелинейньх условий текучести решением задачи (15) или (20) определяются и напряжения. Тогда вектор нагрузки $\mathrm{F}$, характеризуюций огтимальное распределение нагрузки, можно определить из уравнений равновесия. Однако решить задачу (15) при нелинейньх условиг текучести гораздо сложнее, нежели двойственную ей статитескую задачу (24). В случае линейных условий текучести оптимальное распределение нагрузки можно определить только решением математических моделей (24)-(26) статической постановки. В силу указанных обстоятельств предночтительны статические постановки задач, и с практической точки зрения они выходят на первый план.

В гредставленных дискретных математических моделях удовлетворяются дифференциальные уравнения кинематитеской совместности в объеме конечных элемегтов (в ослабленной интегральной форме) и кинематические условия сопряжения межлу элементами, а также кинематические грапичные условия. Поэтому все дискретные математические модели (и кинематические, и статические) будем называть постановками по совместности. Вместе с моделями по равновесию (также в статической и двойственной кинематической формулировках), разработанными в [10] с пргменением равновесньх конечньх элементов, они позволяют 
опреде.тять нижние и верхние оценки распределения предельной нагрузки, в том числе оптимального, и веех параметров напряженнодеформированного состояния тела, отвечающих состоянию простого пластического разрунения. Джя стержневых конструкций эти оценки совпадают п гредставляют собой точное решение. Таким образом, создаются хорошие возможности лия проверки достоверности и точности результатов численныг решений и достаточности густоты конечноэлементной сетки.

Если бы область, занимаемую второй п третьей графами табл. 1, представить, предположим, в виде игрового поля, то разработанные здесь и в [10] математические модели (III группа рабог) позволяют вестп игру численного анализа по всему игровому полю, в то время как в работах первой и второй групп игра ведется только на половине поля. Предлагаемый подход позволяет взглянуть на одну и ту же проблему с четырех сторон.

\section{Jитература}

1. P.G.Hodge, T.Belytschko. Numerical methods for the limit analysis of plates // Trans. ASME, E35, № 4, 1968, p. 796-802.

2. A.Biron, G.Charleux. Limit analysis of axisymmetric pressure vessel intrsections of arbitrary shape // Int. J. Mech. Sci., 14, № 1, 1972, p. 25-41.

3. E.Anderhegeen, H.Knopfel. Finite element analysis using linear programming // Int. J. Solids and Struct., 8, № 12, 1972, p. 1413-1431.

4. S.Turgeman, J.Pastor. Limit analysis: a linear formulation of the kinematic approach for axisymmetric mechanics problems // J. Int. Numer. and Anal. Meth. Geomech., 6, № 1, 1982, p. 109-128.

5. T.Kowai, Y.Toi. A discrete method of limit analysis and its application to plastic stability problems of structural members // Eng. Struct., 5, № 1, 1983, p. 38-44.

6. A.A. Cyras. Analysis and optimization of elastoplastic systems. New York - Chichester - Brisbane - Toronto: Ellis Horwood Limited, 1983. 115 p.

7. A.Čyras and S.Kalanta. Optimal design of cylindrical schels by the finite element technique // Mech. Res. Comm., vol. 1, 1974, p. 125-130.

8. T.Belytschko, M.Velebit. Finite element method for elastic plastic plates // J. Eng. Mech. Div. Proc. Amer. Soc. Civ. Eng., 98, № 1, 1972, p. 227-242.

9. G.Maier, R.A.Zavelani, D.Bennedetti. A finite element approach to optimal design of plastic structures in plane stress // Int. Numer. Meth. Eng., 1972, 4, № 4, p. $455-473$
10. C.Kailahta. Рaвновесные конечно-элементныс постановки запач расчета и оптимизацин предельной нагрузки // Statyba (Строительство), №3(7), Вильнюс: Техника, 1996, с. 13-23

11. С.Каланта. Двойствениые задачи предельного равновесия с разрывами скоростей перемещений // Statyba (Стонтешство), № 2(2), Вильнюс: Техника, 1995, c. $20-25$.

12. АА Чирас. Теория оптимизации в предетьном анализе твертого деформируемого тела. Вильнюс: Мннтис, $1971.123 \mathrm{c}$.

13. А.А.Чирас, А.Э.Боркаускас, Р.П.Каркаускас. Теория и методы оптимизации упруто-пластическнх систем. I : Стройнздат, $1974.280 \mathrm{c}$.

14. Р.Галиагер. Метод конечных элементов. Основы. М.: Мир, 1984. 428 с. (пер. с англ.).

15. С.Каланта. Равновеснье конечные элементы в расчетах упрутих конструкций // Statyba (Строительство), №1(1), Винғюс: Teхника, 1995, с. 25-47.

16. S.Kalanta. Relations and transformations of extremum energy principles for deformable body // Statyba (Civil Engineering), Nr.1(9), Vilnius:Technika, 1997, p.49-56.

Iteikta 19970407

\section{KONSTRUKCIJU RIBINẺS APKROVOS SKAIČIAVIMO UŽDAVINIỤ DUALIEJI MATEMATINIAI MODELIAI NAUDOJANT MIŠRIUOSIUS BAIGTINIUS ELEMENTUS}

\section{S.Kalanta}

Santrauka

Sudaromi standžiojo-plastiškojo kūno ribinès apkrovos analizess ir optimizavimo uždaviniu bendrieji ir diskretieji dualieji matematiniai modeliai kinematine ir statine formuluote, naudojant mišriuosius baigtinius elementus. Juose atsižvelgiama ị energijos disipacijos greiti ne tik baigtinių elementų tūryje, bet ir plastinio tekejimo paviršiuose tarp elementu, kur atsiranda poslinkių greičiu funkciju trūkiai. Taikoma plastinio tekejjimo teorija, dualumo teorija bei matematinis programavimas.

Naudojant straipsnyje [10] pateiktas šių uždavinių bendrąsias statines formuluotes ir Lagranžo daugiklių metodą sudaromi abiejụ uždavinių mišrieji energiniai funkcionalai (1) ir (3). Jų diskretizacijai panaudoti mišrieji baigtiniai elementai. Pasirenkant ittempimul, poslinkiụ greičių, plastiniy daugiklių bei išorinès apkrovos interpoliavimo funkcijas (7) sudaromos mišriujų funkcionalų diskrečiosios išraiškos $(8),(9),(13)$ ir šių funkcionaly stacionarumo pagal statinius kintamuosius (itempimų ir apkrovos vektorius) sąlygos. Iš jų gaunamos geometrinès darnos lygčil bei apkrovos galingumo normalizavimo diskrečiosios išraiškos. Jas naudojant kaip išankstines funkcionalų (8) ir (9) sąlygas sudaromi ribinès apkrovos analizés ir optimizacijos uždavinių kinematinès formuluotes matematiniai modeliai (14), (15), (17). Išeliminavus poslinkil greičius, sudarytas modelis (20) su mažesniu nežinomujų skaičiumi. Naudojant Lagranžo daugikliy metodą sudaryti statines formuluotes matematiniai modeliai (21)-(23) ribinès apkrovos parametro nustatymo uždaviniui ir modeliai (24)-(26) 
apkrovos optimizavimo uždaviniui. Visi jie yra matematinio programavimo uždaviniai.

Inžineriniams tikslams svarbesni ir tinkamesni statinès formuluotes matematiniai modeliai. Jie lengviau išsprendžiami (mažiau nežinomuju), be to, tik jie leidžia nustatyti optimalų apkrovos pasiskirstymą. Sudaryti matematiniai modeliai leidžia rasti ribinès apkrovos, iłtempimų, poslinkių ir plastinių daugikliu greičių viršutines reikšmes. Kartu su šiu uždaviniu pusiausvyriniais modeliais [10], sudarytais naudojant pusiausviruosius baigtinius elementus, jie leidžia sužinoti nurodytų parametrų apatines ir viršutines reikšmes. Strypiniu konstrukciju atveju jos sutampa, t.y. išreiškia tiksluji sprendinị. Taigi atsiranda gera galimybė patikrinti skaitinil skaičiavimo rezultatu patikimumą bei tikslumą ir sužinoti, ar pakankamas baigtinių elementų skaičiuojamojo tinklo tankis.

\section{DUAL MATHEMATICAL MODELS OF LIMIT LOAD ANALYSIS PROBLEMS OF STRUCTURES BY MIXED FINITE ELEMENTS}

\section{S. Kalanta}

\section{Su m m a ry}

The general and discrete dual mathematical models of the limit load analysis and optimization problems of rigid-plastic body are created in the article. The discrete models are formulated by mixed finite elements and presented in terms of kinematic and statiq formulation. In these models the velocity of the energy dissipation is estimated not only within the volume of finite elements, but also at the plastic surfaces between elements, where the discontinuities of displacement velocities functions appear. The theory of plastic flow, the theory of duality and mathematical programming are applied.

The mixed energy functionals (1) and (3) of both problems are formulated using the general static formulations of these problems, presented in the article [10], and Lagrangian multipliers method. The mixed finite elements are used for their discretization. The discrete expressions (8), (9) and (13) of mixed functionals are given choosing the interpolation functions (7) for the stress, displacement velocities, plastic multipliers and external load. Stationary conditions are created by static variables (stress and load vectors) of theses functionals. The discrete expressions of the geometric compatibility equations and constraint of load power are received from them. Using them as preliminary conditions for the functionals (8) and (9), the mathematical models (14), (15) and (17) of kinematic formulation of limit load analysis and optimization problems are formulated. The model (20) with a smaller number of unknowns is formed by elimination the displacement velocities. Using Lagrangian multipliers method, the mathematical models (21)-(23) of static formulation for the limit load parameter analysis problem and the models (24)-(26) for the load optimization problem are derived. All of them are the problems of mathematical programming.

The mathematical models of static formulation for engineering purposes are more important and fit better. They are easier solved (a smaller quantity of unknowns), besides, they allow to determine the optimum distribution of the load. The formulated mathematical models allow to determine upper values of limit load, stresses, displacement and plastic multipliers velocities. Together with equilibrium models of these problems, presented in the article [10], they allow to determine the lower and upper values of aforementioned parameters. So, a good possibility is created to check reliability and exactness of numerical calculation results and to establish, if the computing net density of finite elements is sufficient.

Stanislovas KALANTA. Doctor, Associate Professor. Department of Structural Mechanics. Vilnius Gediminas Technical University, 11 Sauletekio Ave, 2040 Vilnius, Lithuania.

Doctor (structural mechanics), 1974. Research visits to: Leningrad Polytechnic Institute, Moscow Civil Engineering Institute, Kiev Civil Engineering Institute. Research interests: computational mechanics, finite element method, analysis and optimization of elasticplastic structures. 